\title{
ROBUST SENSOR FAULT ESTIMATION FOR DESCRIPTOR-LPV SYSTEMS WITH UNMEASURABLE GAIN SCHEDULING FUNCTIONS: APPLICATION TO AN ANAEROBIC BIOREACTOR
}

\author{
FRANCISCO-RONAY LÓPEZ-ESTRADA ${ }^{a, *}$, JEAN-Christophe PONSART ${ }^{b, c}$, \\ Didier THEILLIOL ${ }^{b, c}$, CARLOS-MANUEL ASTORGA-ZARAGOZA ${ }^{d}$, \\ JORGE-LUIS CAMAS-ANZUETO ${ }^{a}$
}

\author{
${ }^{a}$ Electronic Department, Technological Institute of Tuxtla Gutierrez (ITTG), Tecnológico Nacional de México \\ Carretera Panamericana km 1080, Col. Terán, CP 29050, Tuxtla Gutiérrez, Chiapas, Mexico \\ email $\{$ frlopez, jcamas\} @ittg.edu.mx \\ ${ }^{b}$ CRAN, UMR 7039, University of Lorraine \\ Campus Sciences, B.P. 70239, 54506 Vandoeuvre-lès-Nancy Cedex, France \\ e-mail: \{jean-christophe.ponsart, didier.theilliol\}@univ-lorraine.fr \\ ${ }^{c}$ CNRS, CRAN, UMR 7039, France \\ ${ }^{d}$ National Center of Research and Technological Development (CENIDET), Tecnológico Nacional de México \\ Internado Palmira s/n, Col. Palmira, CP 62490, Cuernavaca, Mor., Mexico \\ e-mail: astorga@cenidet.edu.mx
}

\begin{abstract}
This paper addresses the design of a state estimation and sensor fault detection, isolation and fault estimation observer for descriptor-linear parameter varying (D-LPV) systems. In contrast to where the scheduling functions depend on some measurable time varying state, the proposed method considers the scheduling function depending on an unmeasurable state vector. In order to isolate, detect and estimate sensor faults, an augmented system is constructed by considering faults to be auxiliary state vectors. An unknown input LPV observer is designed to estimate simultaneously system states and faults. Sufficient conditions to guarantee stability and robustness against the uncertainty provided by the unmeasurable scheduling functions and the influence of disturbances are synthesized via a linear matrix inequality (LMI) formulation by considering $H_{\infty}$ and Lyapunov approaches. The performances of the proposed method are illustrated through the application to an anaerobic bioreactor model.
\end{abstract}

Keywords: fault diagnosis, fault estimation, LPV systems, observer design, descriptor system.

\section{Introduction}

Fault detection and isolation (FDI) systems are necessary to ensure the effectiveness of process control, and improve the system's reliability. In general, a fault is something that changes the behaviour of a system such that the system does no longer satisfy its purpose (Lunze et al., 2006). On the other hand, a fault detection and isolation system is related to detection and identification of these changes in order to guarantee both process safety and performance. In the literature this problem has been

\footnotetext{
*Corresponding author
}

addressed by considering various approaches, e.g., parity checks (Gertler, 1997), identification methods (Isermann, 1984), fault detection filters (Wang et al., 2007), among other things; a more detailed review is given by Hwang et al. (2010), Samy et al. (2011), Chen and Patton (1999) or Ding (2008).

Among the available FDI methods, observer-based ones have become most successful techniques. Frequently, these methods address the FDI problem by evaluating the residual signals which contain information about the faults (Frank, 1996). Moreover, a majority of the proposed methods focus on designing FDI systems 
for linear-time invariant (LTI) systems. Nevertheless, it is well known that all systems exhibit nonlinear behavior. In general, the real design of nonlinear fault detection methods is not an easy task, even if the nonlinear system is completely known (Alcorta-García et al., 2014).

More recently, descriptor-linear parameter varying (D-LPV) systems have received increased attention. The main feature of D-LPV systems is to represent the nonlinear dynamics by local linear models which are blended in real time into an overall single model through gain scheduling functions (GSFs). Likewise, in contrast to the classical state-space LPV representation, D-LPV systems are represented by a set of ordinary differential equations (to describe the dynamics) and algebraic ones (to describe interconnections or algebraic constraints). These special attributes of D-LPV systems form a more complete representation of nonlinear systems than state-space LPV systems. In contrast, designing FDI methods for D-LPV systems is more difficult than for state-space ones, because descriptor systems usually have three types of modes: finite dynamic modes, impulsive modes and non-dynamic modes (Duan, 2010). Then, an FDI system should be able to deal with these modes. Applications of descriptor systems can be found in aircraft modelling (Masubuchi et al., 2004), complex systems (Nagy-Kiss et al., 2011a), and electrical, mechanical, or hydraulic systems (Duan, 2010).

Another problem of D-LPV systems is related to gain scheduling functions. Typically, these are designed based on a measurable scheduling vector as the input or the output of the system. Nevertheless, in many applications the scheduling vector could be unmeasurable as the system state. Models which depend on unmeasurable scheduling functions cover a wide class of nonlinear systems, compared with models with measurable scheduling functions. However, the design of control schemes for D-LPV systems with unmeasurable scheduling functions is more difficult than for those with a measurable one. In consequence, few works related to systems with unmeasurable gains scheduling functions have been published. Most of the papers deal with FDI for state-space LPV systems (Yoneyama, 2009; Theilliol and Aberkane, 2011; Ichalal et al., 2010; Chadli et al., 2013a; Blesa et al., 2014). Some of them are related to fault detection for D-LPVsystems with measurable scheduling functions (Hamdi et al., 2012b; Astorga-Zaragoza et al., 2011; Aguilera-González et al., 2013) and only several are related to D-LPV systems. However, we can mention Nagy-Kiss et al. (2011a), who propose a state observer by transforming the D-LPV system with unmeasurable scheduling functions into an equivalent uncertain system. In a work by Nagy Kiss et al. (2011b), an unknown input observer was developed by considering the original system another a perturbed system, where the perturbation vector represents a bounded uncertainty given by measurable and unmeasurable scheduling functions. In both the previous works, the observers were successfully evaluated by using a nonlinear model of a waste-water treatment plant.

In the same context, based on the perturbed system technique, a fault detection scheme was proposed by Hamdi et al. (2012a) with an application to an electrical system. In a work of López-Estrada et al. (2013), the unmeasurable scheduling problem was addressed by considering gain scheduling uncertainties, and designing an $H_{\infty}$ fault estimation observer to be robust against these uncertainties. Even so, robustness against disturbances is not considered. In another attempt (López-Estrada et al., 2014b), an FDI scheme is proposed by considering the uncertain system approach. In this case, robustness against disturbances is examined but no fault estimation.

López-Estrada et al. (2014a) also propose a method based on the convex property of the scheduling functions to obtain an uncertain representation in order to design a robust $H_{\infty}$ observer. It is important to note that in previous works there is no fault reconstruction and the fault detection and isolation are performed by means of normalized residuals generated from a bank of observers. Nevertheless, all these works exemplify the relevance of the techniques for D-LPV systems with unmeasurable scheduling functions and their application to a real process. Nevertheless, due to the lack of research, this problem remains an outstanding and ongoing issue.

The main contribution of this paper is to design a robust state estimation, as well as fault detection, isolation and estimation based on an LPV observer for D-LPV systems with unmeasurable gain scheduling functions. The consideration of unmeasurable scheduling functions is not trivial, since the weighting functions used to synthesize the observer cannot depend on the state variables and, in consequence, it is necessary to estimate them. The research work presented in this article is based on our early work (López-Estrada et al., 2013) with a significant extension consisting in considering additional disturbance rejection to improve state and fault estimation.

In order to solve the unmeasurable scheduling problem, the D-LPV system is transformed into an uncertain one with estimated gain scheduling functions. The fault detection and estimation are solved by considering an augmented system with faults as auxiliary states in the state vector. Consequently, an observer associated with the uncertain augmented system is synthesized to estimate the original states and fault vectors. Sufficient conditions for stability, solvability and robustness are given in terms of LMIs by considering the $H_{\infty}$ technique and Lyapunov theory. The practical contribution of the present paper is application of the proposed method to a realistic model of an anaerobic bioreactor. Simulation results show that the proposed method, compared with the previous work (López-Estrada 
et al., 2013), increases the robustness to the unmeasurable gain scheduling function and disturbances, which are needed to increase reliability and to prevent false alarms.

The problem investigated here is distinguished from most previous literature on D-LPV systems in the following aspects. First, in contrast to the works of Nagy-Kiss et al. (2011a; 2011b), who address only state estimation, the proposed method deals additionally with the FDI problem. The second aspect is related to integrating design of FDI and state estimations. Compared with our previous results (López-Estrada et al., 2014a; 2014b; Hamdi et al. 2012a), the method proposed in this article requires just one observer composed of $h$ models, compared with $h$ observers with $h$ models proposed in the cited papers. This is primarily due to the fact that the method described in this paper does not require a bank of observers to detect faults. Since the number of observers is lower, so is computational complexity, which makes the method attractive for physical implementation. In addition, faults are detected and estimated on time, even in the case of simultaneous faults.

The outline of the paper is as follows. First, the problem formulation is given in Section 2 Sufficient conditions to guarantee stability and robustness on the state and fault estimation observers, as well as some conditions to reduce conservatism and avoid infeasibility numerical solutions are given in Section 3 . Section 4 presents a nonlinear model of the anaerobic bioreactor and its equivalent D-LPV model. Some simulations are presented to illustrate the effectiveness of the proposed method. Finally, some concluding remarks are given in Section 5

The notation used in this article is standard. For a matrix $A \in \mathbb{R}^{n \times n}, A^{T}, A^{-1}$ and $A^{\dagger}$ denote its transpose, inverse and pseudoinverse, respectively. $\operatorname{He}\{A\}$ is a shorthand notation for $A+A^{T}$. The asterisk $\star$ denotes the transposed elements in the symmetric positions of a matrix.

\section{Problem formulation}

Consider a descriptor LPV system under sensor faults and disturbances given by

$$
\begin{aligned}
\tilde{E} \dot{\tilde{x}}(t) & =\sum_{i=1}^{h} \rho_{i}(\tilde{x}(t))\left[\tilde{A}_{i} \tilde{x}(t)+\tilde{B}_{i} u(t)+\tilde{B}_{d} d(t)\right] \\
y(t) & =\tilde{C} \tilde{x}(t)+\tilde{D}_{d} d(t)+f(t)
\end{aligned}
$$

where $\tilde{x}(t) \in \mathbb{R}^{n}, u(t) \in \mathbb{R}^{m}, d(t) \in \mathbb{R}^{l}, y(t) \in \mathbb{R}^{p}$, and $f(t) \in \mathbb{R}^{p}$ are the state vector, the control input, the disturbance vector, the measured vector and the sensor fault vector, respectively. $\tilde{A}_{i}, \tilde{B}_{i}, \tilde{B}_{d}, \tilde{C}, \tilde{D}_{d}$ are constant matrices of appropriate dimensions, $\operatorname{rank}(\tilde{E})=r \leq n$. $h$ is the number of models and $\rho_{i}(\tilde{x}(t))$ 's are the scheduling functions which are considered to be depending on the unmeasurable state vector $\tilde{x}(t)$. The scheduling function satisfies the following convex property:

$$
\mathscr{F}(t):=\left\{\begin{array}{l}
\forall i \in 1,2, \ldots, h, \rho_{i}(\tilde{x}(t)) \geq 0, \\
\sum_{i=1}^{h} \rho_{i}(\tilde{x}(t))=1, \forall t .
\end{array}\right.
$$

In order to estimate the states and sensor faults, we considered faults as an auxiliary state of the augmented state system

$$
x(t)=\left[\begin{array}{ll}
\tilde{x}^{T}(t) & f^{T}(t)
\end{array}\right]^{T},
$$

such that the system (1) becomes

$$
\begin{aligned}
E \dot{x}(t)= & \sum_{i=1}^{h} \rho_{i}\left[A_{i} x(t)+B_{i} u(t)\right. \\
& \left.+B_{d} d(t)+B_{f} f(t)\right], \\
y(t)= & C x(t)+D_{d} d(t),
\end{aligned}
$$

where

$$
\begin{array}{rlrl}
E & =\left[\begin{array}{cc}
\tilde{E} & 0 \\
0 & 0_{p}
\end{array}\right], & A_{i}=\left[\begin{array}{cc}
\tilde{A}_{i} & 0 \\
0 & -I_{p}
\end{array}\right], \\
B_{i}=\left[\begin{array}{c}
\tilde{B}_{i} \\
0_{p}
\end{array}\right] & B_{d}=\left[\begin{array}{c}
\tilde{B}_{d} \\
0
\end{array}\right], \\
B_{f}=\left[\begin{array}{c}
0 \\
I_{p}
\end{array}\right], & C=\left[\begin{array}{ll}
\tilde{C} & I_{p}
\end{array}\right], \\
D_{d}=\tilde{D}_{d} . &
\end{array}
$$

For this system, the stability condition is given by the following lemma:

Lemma 1. (Chadli and Darouach, 2011) The system (3) is said to be stable if there exists a Lyapunov function $V(x(t))=x^{T}(t) E^{T} P x(t)$, where $E^{T} P=$ $P^{T} E \geq 0$, whose derivative $\dot{V}(x(t))=\dot{x}^{T}(t) E^{T} P x(t)+$ $x^{T} E^{T} P \dot{x}(t)$ is negative.

The following assumptions express observability properties of D-LPV systems

Assumption 1. (Hamdi et al., 2012) The system (3) and the triple $\left(E, A_{i}, C\right)$ are called observable on the reachable set ( $\mathrm{R}$-observable) if

$$
\operatorname{rank}\left[\begin{array}{c}
s E-A_{i} \\
C
\end{array}\right]=n, \quad \forall i \in\{1,2, \ldots, h\} .
$$

Assumption 2. (Hamdi et al., 2012) The system (3) and the triple $\left(E, A_{i}, C\right)$ are called impulse observable (I-observable) if

$$
\begin{aligned}
\operatorname{rank}\left[\begin{array}{cc}
E & A_{i} \\
0 & E \\
0 & C
\end{array}\right]=n+\operatorname{rank} E, & \\
& \forall i \in\{1,2, \ldots, h\} .
\end{aligned}
$$


Remark 1. R-observability characterizes the ability to reconstruct only the reachable state from the output data. However, due to algebraic equations, impulsive terms can appear. These are not desirable since they can saturate the state response or, in general, they can have negative effects on the system. I-observability guarantees the ability to estimate impulse terms given by the algebraic equations. Note that observers for D-LPV systems with unmeasurable scheduling functions are designed such that each scheduling function has a local gain. Therefore it is required that the local models be observable or detectable. However, due to the form of the observer (6), it is required to assume that the local models are $\mathrm{R} / \mathrm{I}$ observable (Kamidi, 2000; Lendek et al., 2011).

With the assumptions that the D-LPV system is $\mathrm{R} / \mathrm{I}$-observable, the following LPV observer is proposed:

$$
\begin{aligned}
& \dot{z}(t)=\sum_{j=1}^{h} \rho_{j}(\hat{x}(t))\left[N_{j} z(t)+G_{j} u(t)+L_{j} y(t)\right], \\
& \hat{x}(t)=z(t)+T_{2} y(t)
\end{aligned}
$$

where $z(t)$ is an auxiliary state vector, $\hat{x}(t)$ is the state estimate, $N_{j}, G_{j}, L_{j}$ and $T_{2}$ are gain matrices of appropriate dimensions, while $\rho_{j}(\hat{x}(t))$ are convex gain scheduling functions which are considered to be dependent on the estimated time varying state $\hat{x}(t)$. Additionally, an auxiliary residual vector is defined as

$$
r(t)=y(t)-C \hat{x}(t)
$$

The problem of fault estimation is reduced to finding the gain matrices of the observer (6) which maximize robustness to the unmeasurable scheduling function $\rho_{i}(x(t))$ such that $\lim _{t \rightarrow \infty}|e(t)| \approx \lim _{t \rightarrow \infty} \mid x(t)-$ $\hat{x}(t) \mid \approx 0$. In addition, the effects of disturbances must be also attenuated.

\section{Observer design}

As already mentioned, the system (3) is dependent on the unmeasurable state $x(t)$ and the observer is dependent on the estimated state $\hat{x}(t)$. Therefore, in order to synthesize the observer gains, it is necessary to obtain a system which considers both scheduling functions. To solve this problem, the system (3) is transformed into an uncertain one by adding and subtracting the term $\sum_{j=1}^{h} \rho_{j}(\hat{x}(t))\left(A_{j} x(t)+B_{j} u(t)\right)$. After algebraic manipulations, the original system (3) becomes

$$
\begin{aligned}
E \dot{x}(t)= & \sum_{i, j=1}^{h} \rho_{i} \hat{\rho}_{j}\left[A_{i j} x(t)+B_{i j} u(t)+B_{d} d(t)\right. \\
& \left.+B_{f} f(t)\right], \\
y(t)= & C x(t)+D_{d} d(t),
\end{aligned}
$$

where

$$
\begin{aligned}
\sum_{i, j=1}^{h} \rho_{i} \hat{\rho}_{j} & =\sum_{i=1}^{h} \sum_{j=1}^{h} \rho_{i}(x(t)) \rho_{j}(\hat{x}(t)), \\
A_{i j} & =A_{j}+\Delta A_{i j}, \quad \Delta A_{i j}=A_{i}-A_{j}, \\
B_{i j} & =B_{j}+\Delta B_{i j}, \quad \Delta B_{i j}=B_{i}-B_{j} .
\end{aligned}
$$

Note that the previous transformation is possible due to the convex property of the scheduling functions which considers $\sum_{i=1}^{h} \rho_{i}(x(t))=\sum_{i=1}^{h} \rho_{j}(\hat{x}(t))=1$.

The estimation error is given as

$$
\begin{aligned}
& e(t)=x(t)-\hat{x}(t), \\
& e(t)=\left(I-T_{2} C\right) x(t)-z(t)-T_{2} D_{d} d(t),
\end{aligned}
$$

assuming that there exists $T_{1} \in \mathbb{R}^{n \times n}$ such that

$$
T_{1} E=I-T_{2} C
$$

A particular solution of matrices $T_{1}$ and $T_{2}$ is computed as

$$
\left[\begin{array}{ll}
T_{1} & T_{2}
\end{array}\right]=\left[\begin{array}{l}
E \\
C
\end{array}\right]^{\dagger} .
$$

Consequently, the error equation is given by

$$
e(t)=T_{1} E x(t)-z(t)-T_{2} D_{d} d(t) .
$$

In order to eliminate the influence of $d(t)$ in 13, it is assumed that unknown inputs are of slow variation, i.e, $\dot{d}(t) \approx 0$. From a practical point of view, this condition can be relaxed as discussed by Hamdi et al. (2012b) and Chadli et al. (2013b).

The dynamics of the error equation are rewritten by considering the slow variation condition as

$$
\begin{aligned}
\dot{e}(t)= & T_{1} E \dot{x}(t)-\dot{z}(t) \\
\dot{e}(t)= & \sum_{i, j=1}^{h} \rho_{i} \hat{\rho}_{j}\left[T _ { 1 } \left(A_{i j} x(t)+B_{i j} u(t)+B_{d} d(t)\right.\right. \\
& \left.\left.+B_{f} f(t)\right)-\left(N_{j} z(t)+G_{j} u(t)+L_{j} y(t)\right)\right] \\
\dot{e}(t)= & \sum_{i, j=1}^{h} \rho_{i} \hat{\rho}_{j}\left[\left(T_{1} A_{j}-L_{j} C-N_{j} T_{1} E\right) x(t)\right. \\
& +T_{1} \Delta A_{i j} x(t)+\left(T_{1} B_{j}-G_{j}\right) u(t) \\
& +T_{1} \Delta B_{i j} u(t)+\left(T_{1} B_{d}-L_{j} D_{d}\right) d(t) \\
& \left.+T_{1} B_{f} f(t)+N_{j} e(t)\right]
\end{aligned}
$$

assuming that

$$
\begin{aligned}
T_{1} A_{j}-L_{j} C-N_{j} T_{1} E & =0, \\
G_{j}-T_{1} B_{j} & =0 .
\end{aligned}
$$


By manipulating (16), the following expressions are equivalent:

$$
\begin{array}{ll}
N_{j}=T_{1} A_{j}+K_{j} C, & \forall j \in\{1,2, \ldots, h\}, \\
K_{j}=N_{j} T_{2}-L_{j}, & \forall j \in\{1,2, \ldots, h\} .
\end{array}
$$

By substituting these conditions in 15 and considering (7), the state-space residual equation is obtained as

$$
\begin{aligned}
\dot{e}(t)= & \sum_{i, j=1}^{h} \rho_{i} \hat{\rho}_{j}\left[N_{j} e(t)+T_{1} \Delta A_{i j} x(t)+T_{1} \Delta B_{i j} u(t)\right. \\
& \left.+\left(T_{1} B_{d}+K_{j} D_{d}\right) d(t)+T_{1} B_{f} f(t)\right] \\
r(t)= & C e(t)+D_{d} d(t) .
\end{aligned}
$$

Then a standard representation is arrived at by considering the augmented states $x_{e}(t)=\left[e(t)^{T} x(t)^{T}\right]^{T}$ such as

$$
\begin{aligned}
\bar{E} x_{e}(t) & =\bar{A} x_{e}(t)+\bar{B} \bar{f}_{d}(t), \\
r(t) & =\bar{C} x_{e}(t)+\bar{D}_{d} \bar{f}_{d}(t),
\end{aligned}
$$

where

$$
\begin{aligned}
\bar{E} & =\left[\begin{array}{ll}
I & 0 \\
0 & E
\end{array}\right], \quad \bar{A}=\sum_{i, j=1}^{h} \rho_{i} \hat{\rho}_{j}\left[\begin{array}{cc}
N_{j} & T_{1} \Delta A_{i j} \\
0 & A_{i}
\end{array}\right], \\
\bar{B} & =\sum_{i, j=1}^{h} \rho_{i} \hat{\rho}_{j}\left[\begin{array}{ccc}
T_{1} \Delta B_{i j} & T_{1} B_{d}+K_{j} D_{d} & T_{1} B_{f} \\
B_{i} & B_{d} & B_{f}
\end{array}\right], \\
\bar{C} & =\left[\begin{array}{ll}
C & 0
\end{array}\right], \quad \bar{D}_{d}=\left[\begin{array}{lll}
0 & \tilde{D}_{d} & 0
\end{array}\right], \\
\bar{f}_{d} & =\left[\begin{array}{lll}
u(t) d(t) f(t)
\end{array}\right]^{T} .
\end{aligned}
$$

Equivalently, the transfer from the input $\bar{f}_{d}$ to $r(t)$ is written as

$$
G_{r \bar{f}_{d}}=\left\{\bar{E},\left[\begin{array}{c|c}
\bar{A} & \bar{B} \\
\hline \bar{C} & \bar{D}_{d}
\end{array}\right]\right\} .
$$

Sufficient conditions to guarantee robustness to unknown inputs and the error provided by the unmeasurable scheduling function such that the norm $\left\|G_{r \bar{f}_{d}}\right\|_{\infty} \leq \gamma$ are given by the following theorem:

Theorem 1. Consider the system (1), the augmented system (3), and the observer (6). Let the attenuation level satisfy $\gamma>0$. The quadratic stability of the estimation error is guaranteed if $\left\|G_{r \bar{f}_{d}}\right\|_{\infty}<\gamma$ and if there exist matrices

$$
P=\left[\begin{array}{cc}
P_{1} & 0 \\
0 & P_{2}
\end{array}\right], \quad P_{1}=P_{1}^{T}>0,
$$

such that the following optimization problem holds $\forall i, j \in$ $\{1,2, \ldots, h\}$ :

$$
\min _{P_{1}, P_{2}, Q_{i}} \gamma
$$

subject to

$$
E^{T} P_{2}=P_{2}^{T} E \geq 0
$$

$$
\left[\begin{array}{cccccc}
\Phi_{11} & \Phi_{12} & P_{1} T_{1} \Delta B_{i j} & \Phi_{14} & P_{1} T_{1} B_{f} & C^{T} \\
\star & \Phi_{22} & P_{2} B_{i} & P_{2} B_{d} & P_{2} B_{f} & 0 \\
\star & \star & -\gamma^{2} I & 0 & 0 & 0 \\
\star & \star & \star & -\gamma^{2} I & 0 & D_{d}^{T} \\
\star & \star & \star & \star & -\gamma^{2} I & 0 \\
\star & \star & \star & \star & \star & -I
\end{array}\right]<0,
$$

where

$$
\begin{aligned}
& \Phi_{11}=\operatorname{He}\left\{\left(T_{1} A_{j}\right)^{T} P_{1}+Q_{j} C\right\} \\
& \Phi_{12}=P_{1}\left(T_{1} \Delta A_{i j}\right) \\
& \Phi_{14}=P_{1} T_{1} B_{d}+Q_{j} D_{d}, \\
& \Phi_{22}=\operatorname{He}\left\{A_{i}^{T} P_{2}\right\}
\end{aligned}
$$

with $T_{1}$ given by

$$
\left[\begin{array}{ll}
T_{1} & T_{2}
\end{array}\right]=\left[\begin{array}{l}
E \\
C
\end{array}\right]^{\dagger}
$$

Then, the gain matrices of the observer (6) are given by $K_{j}=P_{1}^{-1} Q_{j}$ and the equations defined in (17)-(19).

Proof. In order to provide residual signals robust to the unmeasurable scheduling functions, $H_{\infty}$ performance can be guaranteed by considering the following criterion:

$$
\begin{aligned}
J_{r \bar{f}_{d}}:= & \int_{0}^{\infty} r^{T}(t) r(t) \mathrm{d} \tau \\
& -\gamma^{2} \int_{0}^{\infty} \bar{f}_{d}^{T}(t) \bar{f}_{d}(t) \mathrm{d} \tau<0 .
\end{aligned}
$$

Note that these performance criteria guaranteed disturbance and uncertainties attenuation in relation to $\gamma$. Additionally, asymptotic stability is reached by considering a Lyapunov function $V\left(x_{e}(t)\right)=\Omega(t)=x_{e}^{T}(t) \bar{E}^{T} P x_{e}(t)$, such that the criteria performance can be manipulated as

$$
\begin{aligned}
J_{r \bar{f}_{d}} & \\
:= & \left.\left.\int_{0}^{\infty}\left(r^{T}(t) r(t)-\gamma^{2} \bar{f}_{d}^{T}(t) \bar{f}_{d}(t)\right)+\dot{\Omega}(t)\right)\right) \mathrm{d} \tau \\
& -\Omega(t)<0 \\
= & \int_{0}^{\infty}\left(x_{e}^{T}(t) \bar{C}^{T} \bar{C} x_{e}(t)+x_{e}^{T}(t) \bar{C}^{T} \bar{D}_{d} \bar{f}_{d}(t)\right. \\
& +\bar{f}_{d}^{T}(t) \bar{D}_{d}^{T} \bar{C} x_{e}(t)+\bar{f}^{T}(t) \bar{D}_{d}^{T} \bar{D}_{d} \bar{f}_{d}(t) \\
& \left.\left.\left.-\gamma^{2} \bar{f}_{d}^{T}(t) \bar{f}_{d}(t)+\dot{\Omega}(t)\right) \mathrm{d} \tau-\Omega(t)\right)\right)<0 .
\end{aligned}
$$


For given matrices $\bar{C}$ and $\bar{D}_{d}$ from the extended states of (21), the following expression is obtained:

$$
\left.\left.\int_{0}^{\infty}\left(\left[\begin{array}{l}
e(t) \\
x(t) \\
u(t) \\
d(t) \\
f(t)
\end{array}\right]^{T} \Gamma\left[\begin{array}{l}
e(t) \\
x(t) \\
u(t) \\
d(t) \\
f(t)
\end{array}\right]+\dot{\Omega}(t)\right] \mathrm{d} \tau-\Omega(t)\right)\right)<0
$$

with

$$
\Gamma=\left[\begin{array}{ccccc}
C^{T} C & 0 & 0 & C^{T} D_{d} & 0 \\
0 & 0 & 0 & 0 & 0 \\
0 & 0 & -\gamma^{2} I & 0 & 0 \\
D_{d}^{T} C & 0 & 0 & D_{d}^{T} D_{d}-\gamma^{2} I & 0 \\
0 & 0 & 0 & 0 & -\gamma^{2} I
\end{array}\right]
$$

The dynamics of the Lyapunov equation $\Omega(t)$ are manipulated as

$$
\dot{\Omega}(t)=\dot{x}_{e}^{T}(t) \bar{E}^{T} P x_{e}(t)+x_{e}^{T} \bar{E}^{T} P \dot{x}_{e}(t)
$$

and by considering $\bar{E}^{T} P=P^{T} \bar{E}<0$. Then

$$
\begin{aligned}
\dot{\Omega}(t)= & \dot{x}_{e}^{T}(t) \bar{E}^{T} P x_{e}(t)+x_{e}^{T} \bar{E}^{T} P \dot{x}_{e}(t) \\
= & x_{e}^{T}(t) \bar{A}^{T} P x_{e}(t)+\bar{f}^{T}(t) \bar{B}^{T} P x_{e}(t) \\
& +x_{e}^{T}(t) P^{T} \bar{A} x_{e}(t)+x_{e}^{T}(t) P^{T} \bar{B} \bar{f}(t) \\
= & {\left[\begin{array}{c}
x_{e} \\
\bar{f}(t)
\end{array}\right]^{T}\left[\begin{array}{cc}
\bar{A}^{T} P+P^{T} \bar{A} & P^{T} \bar{B} \\
\star & 0
\end{array}\right]\left[\begin{array}{c}
x_{e} \\
\bar{f}(t)
\end{array}\right] . }
\end{aligned}
$$

Here

$$
P=\left[\begin{array}{cc}
P_{1} & 0 \\
0 & P_{2}
\end{array}\right]
$$

and $\bar{E}^{T} P=P^{T} \bar{E} \geq 0$ are manipulated as

$$
\left[\begin{array}{cc}
P_{1} & 0 \\
0 & E^{T} P_{2}
\end{array}\right]=\left[\begin{array}{cc}
P_{1}^{T} & 0 \\
0 & P_{2}^{T} E
\end{array}\right] \geq 0 .
$$

It can be seen that $P_{1}=P_{1}^{T} \geq 0$ and $E^{T} P_{2}=P_{2}^{T} E \geq 0$. The term $\bar{A}^{T} P+P^{T} \bar{A}$ can be manipulated as

$$
\left[\begin{array}{cc}
\mathrm{He}\left\{\left(T_{1} A_{j}\right)^{T} P_{1}+Q_{j} C\right\} & P_{1}\left(T_{1} \Delta A_{i j}\right) \\
\star & \operatorname{He}\left\{A_{i}^{T} P_{2}\right\}
\end{array}\right]<0 .
$$

By considering the extended matrices from (21), the Lyapunov equation (28) is rewritten as

$$
\begin{aligned}
& \dot{\Omega}(t)=\sum_{i, j=1}^{h} \rho_{i} \hat{\rho}_{j}\left[\begin{array}{l}
e(t) \\
x(t) \\
u(t) \\
d(t) \\
f(t)
\end{array}\right]^{T}\left[\begin{array}{cc}
\Phi_{11} & P_{1}\left(T_{1} \Delta A_{i j}\right) \\
\star & \operatorname{He}\left\{A_{i}^{T} P_{2}\right\} \\
\star & \star \\
\star & \star \\
\star & \star
\end{array}\right. \\
& \left.P_{1} T_{1} \Delta B_{i j} \quad \Phi_{14} \quad P_{1} T_{1} B_{f}\right]\left[\begin{array}{l}
e(t) \\
e(t)
\end{array}\right. \\
& \left.\begin{array}{ccc}
P_{2}^{T} B_{i} & P_{2}^{T} B_{d} & P_{2}^{T} B_{f}
\end{array}\right] x(t) \\
& \left.\begin{array}{ccc}
0 & 0 & 0 \\
\star & 0 & 0 \\
\star & \star & 0
\end{array}\right]\left[\begin{array}{l}
u(t) \\
d(t) \\
f(t)
\end{array}\right]
\end{aligned}
$$
obtain

Then, by substituting $\dot{\Omega}(t)$ in the criteria $J_{r \bar{f}_{d}}$, we

$$
\begin{aligned}
J_{r \bar{f}_{d}}= & \int_{0}^{\infty} \sum_{i, j=1}^{h} \rho_{i} \hat{\rho}_{j}\left[\begin{array}{l}
e(t) \\
x(t) \\
u(t) \\
f(t)
\end{array}\right]^{T} \Theta\left[\begin{array}{l}
e(t) \\
x(t) \\
u(t) \\
f(t)
\end{array}\right] \mathrm{d} t \\
& -V\left(x_{e}(t)\right)<0,
\end{aligned}
$$

with

$$
\begin{gathered}
\Theta=\left[\begin{array}{ccc}
\Phi_{11}+C^{T} C & P_{1}\left(T_{1} \Delta A_{i j}\right) & P_{1} T_{1} \Delta B_{i j} \\
\star & \operatorname{He}\left\{A_{i}^{T} P_{2}\right\} & P_{2}^{T} B_{i} \\
\star & \star & -\gamma^{2} I \\
\star & \star & \star \\
\star & \star & \star \\
\Phi_{14}+C^{T} D_{d} & P_{1} T_{1} B_{f} \\
P_{2}^{T} B_{d} & P_{2}^{T} B_{f} \\
0 & 0 \\
D_{d}^{T} D_{d}-\gamma^{2} I & 0 \\
\star & -\gamma^{2} I
\end{array}\right]<0 .
\end{gathered}
$$

It can be noticed that, if $\Theta<0$, then $J_{r \bar{f}_{d}}<0$. The Schur complement implies (24). Finally, in order to guarantee an optimal solution for $\gamma$, the problem is reformulated as an optimization one as written in Theorem 1 This completes the proof.

3.1. Feasibility and gain synthesis. Numerical problems or infeasible solutions can appear due to the singular form of the equality constraint (23) in Theorem 1 The following lemma provides a sufficient condition to transform the equality constraint $E^{T} P_{2}=P_{2}^{T} E$ into the LMI form.

Lemma 2. (Xu and Lam, 2006) All $Z \in \mathbb{R}^{n \times n}$ satisfying

$$
E^{T} Z=Z^{T} E \geq 0
$$

can be parametrized as

$$
\mathscr{Z}=Z E+S X
$$

where $Z>0 \in \mathbb{R}^{n \times n}$ and $X \in \mathbb{R}^{(n-r) \times n}$ are parameter matrices. $S \in \mathbb{R}^{n \times(n-r)}$ is any matrix with full column rank and satisfies $E^{T} S=0$.

From Lemma 2, the following result is obtained.

Corollary 1. Consider the system (1), the augmented system (3), and the observer (6). Let the attenuation level satisfy $\gamma>0$. The quadratic stability of the estimation error is guaranteed if $\left\|G_{r \bar{f} d}\right\|_{\infty}<\gamma$ and if there exist matrices

$$
P=\left[\begin{array}{cc}
P_{1} & 0 \\
0 & \mathscr{P}_{2}
\end{array}\right], \quad P_{1}=P_{1}^{T}>0
$$




$$
\mathscr{P}_{2}=P_{2} E+S X, \quad P_{2}>0, X \in \mathbb{R}^{(n-r) \times n},
$$

such that the following optimization problem holds, $\forall i, j \in\{1,2, \ldots, h\}$ :

$$
\min _{P_{1}, P_{2}, Q_{i}, X} \gamma
$$

subject to

$$
\left[\begin{array}{cccc}
\Phi_{11} & \Phi_{12} & P_{1} T_{1} \Delta B_{i j} & \Phi_{14} \\
\star & \Phi_{22} & \mathscr{P}_{2}^{T} B_{i} & \mathscr{P}_{2}^{T} B_{d} \\
\star & \star & -\gamma^{2} I & 0 \\
\star & \star & \star & -\gamma^{2} I \\
\star & \star & \star & \star \\
\star & \star & \star & \star \\
& & P_{1} T_{1} B_{f} & C^{T} \\
& & \mathscr{P}_{2}^{T} B_{f} & 0 \\
& & 0 & 0 \\
& & 0 & D_{d}^{T} \\
& & -\gamma^{2} I & 0 \\
& & \star & -I
\end{array}\right]<0,
$$

with

$$
\Phi_{22}=\operatorname{He}\left\{A_{i}^{T} \mathscr{P}_{2}\right\}
$$

where $T_{1}$ is given by

$$
\left[\begin{array}{ll}
T_{1} & T_{2}
\end{array}\right]=\left[\begin{array}{l}
E \\
C
\end{array}\right]^{\dagger}
$$

$S \in \mathbb{R}^{n \times(n-r)}$ is any matrix with full column rank which satisfies $E^{T} S=0$. Then the gain matrices of the observer (6) are given by $K_{j}=P_{1}^{-1} Q_{j}$ and the equations defined in (17)-(19).

Proof. Sufficiency can be easily proved by substituting $\mathscr{P}_{2}=P_{2} E+S X$ in $(23)$, and with the condition $E^{T} S=$ 0 it is easy to prove that $E^{T} P_{2}=P_{2}^{T} E \geq 0$.

\section{Application: An anaerobic bioreactor}

The proposed method is evaluated via numerical simulations by using a fourth-order mathematical model which has been previously described and validated by Martínez-Sibaja et al. (2011). This model represents an upflow anaerobic sludge blanket bioreactor (UASB). The state variables are $\tilde{x}_{1}(t)=x o(t)$, the concentration of the anaerobic biomass; $\tilde{x}_{2}(t)=s_{1}(t)$, the concentration of organic matter expressed as chemical oxygen demand (COD); $\tilde{x}_{3}(t)=Q_{C H 4}(t)$, the outlet flux of methane bio-gas, and $\tilde{x}_{4}(t)=\mu(t)$, the specific growth rate. The inputs variables are: $u_{1}(t)=D(t)$, the dilution rate, and $u_{2}(t)=s_{1}^{i}(t)$, the concentration of COD in the yield affluent. From the model given by Martínez-Sibaja et al. (2011), the following nonlinear descriptor system is deduced:

$$
\begin{aligned}
\dot{\tilde{x}}(t) & =f_{1}(\tilde{x}(t), u(t)), \\
0 & =f_{2}(\tilde{x}(t), u(t)),
\end{aligned}
$$

with

$$
\begin{aligned}
& f_{1}(\tilde{x}(t), u(t)) \\
& \quad=\left[\begin{array}{c}
Y_{1} \mu(t) x_{o}(t)-\alpha D(t) x_{o}(t)-k_{d} x_{o}(t) \\
D(t)\left(s_{1}(t)^{i}-s_{1}(t)\right)-\mu(t) x_{o} \\
\left(1-Y_{1}\right) Y_{C H 4} \mu(t) x_{o}-Q_{C H 4}(t)
\end{array}\right], \\
& f_{2}(\tilde{x}(t), u(t))=k_{m 1} \frac{s_{1}(t)}{k_{s 1}+s_{1}(t)} I_{p H}-\mu(t),
\end{aligned}
$$

where $k_{m l}, k_{d}, k_{s 1}$ are the specific growth rates of mass, the dilution rate of the anaerobic reactor and the constant decrease in semi-saturation for the biomass, respectively. $Y_{1}$ is the coefficient of degradation of COD, $I_{p H}$ represents the $p H$ inhibition, where $p H_{L L}$ and $p H_{U L}$ are the lower and higher $p H$ limits. The values of the constants parameters are shown in Table 1 The process outputs are $y_{1}(t)=x_{o}, y_{2}(t)=s_{1}(t)$ and $y_{3}(t)=Q_{C H 4}$.

Table 1. Model parameters.

\begin{tabular}{|l|l|}
\hline Parameter & Value \\
\hline \hline$k_{m 1}$ & $5.1 \mathrm{gCOD} / \mathrm{gCOD} \mathrm{d}$ \\
$k_{s 1}$ & $0.5 \mathrm{gCOD} / 1$ \\
$k_{d}$ & $0.02 \mathrm{1} / \mathrm{d}$ \\
$Y_{1}$ & $0.1 \mathrm{gCOD} / \mathrm{g} \mathrm{COD}$ \\
$Y_{C H 4}$ & $0.351_{C H 4} / \mathrm{g} \mathrm{COD}$ \\
$\alpha$ & 0.5 \\
$I_{p H}$ & 0.9068 \\
\hline
\end{tabular}

The nonlinear descriptor system given by (36) and (37) can be written as

$$
\begin{aligned}
{\left[\begin{array}{c}
\dot{\tilde{x}}_{1}(t) \\
\dot{\tilde{x}}_{2}(t) \\
\tilde{\tilde{x}}_{3}(t) \\
0
\end{array}\right]=} & {\left[\begin{array}{ccc}
-k_{d} & 0 & 0 \\
0 & 0 & 0 \\
0 & 0 & -1 \\
0 & \frac{k_{m 1}}{k_{s 1}+\tilde{x}_{2}(t)} I_{p H} & 0 \\
Y_{1} \tilde{x}_{1}(t) \\
-\tilde{x}_{1}(t) \\
-1 \\
-1
\end{array}\right]\left[\begin{array}{c}
\tilde{x}_{1}(t) \\
\tilde{x}_{2}(t) \\
\tilde{x}_{3}(t) \\
\tilde{x}_{4}(t)
\end{array}\right] } \\
& \left(1-Y_{1}\right) Y_{C H 4} \tilde{x}_{1}(t) \\
+ & {\left[\begin{array}{cc}
-\alpha \tilde{x}_{1}(t) & 0 \\
-\tilde{x}_{2}(t) & u_{1}(t) \\
0 & 0 \\
0 & 0
\end{array}\right]\left[\begin{array}{l}
u_{1}(t) \\
u_{2}(t)
\end{array}\right] . }
\end{aligned}
$$

In order to obtain a D-LPV representation, the scheduling variables are chosen as $\underline{a}_{k}<\zeta(t)<\bar{a}_{k}, \forall k=$ $1,2, \ldots, 4$, where $k$ is the number of non-constant elements in the system (38). The minimum and maximum 
bounded limits $\underline{a}_{k}$ and $\bar{a}_{k}$ for $k=1, \ldots, 4$, are selected according to experimental constraints as given by Martínez-Sibaja et al. (2011),

$$
\begin{aligned}
\zeta_{1} & =\tilde{x}_{1}(t) \in\left[\bar{a}_{1}, \underline{a}_{1}\right]=\left[\begin{array}{ll}
0.2 & 0.7
\end{array}\right], \\
\zeta_{2} & =\tilde{x}_{2}(t) \in\left[\bar{a}_{2}, \underline{a}_{2}\right]=\left[\begin{array}{ll}
0.01 & 0.6
\end{array}\right], \\
\zeta_{3} & =u_{1}(t) \in\left[\bar{a}_{3}, \underline{a}_{3}\right]=\left[\begin{array}{ll}
0.2 & 0.8
\end{array}\right], \\
\zeta_{4} & =\frac{k_{m 1}}{k_{s 1}+\tilde{x}_{2}(t)} I_{p H} \in\left[\bar{a}_{4}, \underline{a}_{4}\right]=\left[\begin{array}{ll}
3.7 & 9.9
\end{array}\right] .
\end{aligned}
$$

For each $\zeta_{j}$, two local scheduling functions are constructed as

$$
\mu_{1}^{k}\left(\zeta_{k}\right)=\frac{\overline{a_{k}}-\zeta_{k}}{\bar{a}_{k}-\underline{a}_{k}}, \quad \mu_{2}^{k}=1-\mu_{1}^{k}, \quad k=1, \ldots, 4 .
$$

These two weighting functions are normalized such that $\mu_{1}^{k}>0, \mu_{2}^{k}>0$, and $\mu_{1}^{k}+\mu_{2}^{k}=1$ for any value of $z_{k}$. Therefore, for $k=4,2^{4}=16$ scheduling functions are computed as the product of the weighting functions that correspond to each local model

$$
\rho_{i}(\zeta(t))=\prod_{k=1}^{4} \mu_{i k}\left(\zeta_{i}\right)
$$

where $\mu_{i k}$ is either $\mu_{1}^{k}$ or $\mu_{2}^{k}$, depending on which local weighting function is considered.

The scheduling functions are normal $\rho_{i}(\zeta(t)) \geq$ $0, \sum_{i=1}^{16} \rho_{i}(\zeta(t))=1$.

Remark 2. Without loss of generality, we change the notation from $\rho_{i}(x(t))$ to $\rho_{i}(\zeta(t))$, because in this particular example there are two nonlinear terms related to the states and one to the input. Note also that, although the scheduling equations are well known as given in (40), the scheduling vector $\zeta(t)$ is considered unmeasurable and needs to be estimated. Therefore, for the observer implementation the scheduling function $\rho_{i}(\zeta(t))$ is expressed as $\rho_{j}(\hat{\zeta}(t))$, which indicates the estimation of the unmeasurable states $\tilde{x}_{1}(t), \tilde{x}_{2}(t)$ and the output $u(t)$.

By considering the scheduling variables on the nonlinear matrix (38), a descriptor quasi-LPV model is obtained as

$$
\begin{aligned}
E \dot{\tilde{x}}(t) & =\sum_{i=1}^{8} \rho_{i}(\zeta(t))\left[\tilde{A}_{i} \tilde{x}(t)+\tilde{B}_{i} u(t)\right], \\
y(t) & =\tilde{C} \tilde{x}(t),
\end{aligned}
$$

$$
\begin{aligned}
& \tilde{E}=\operatorname{diag}(1,1,1,0), \\
& \tilde{A}_{i}=\left[\begin{array}{cccc}
-k_{d} & 0 & 0 & Y_{1} \zeta_{1} \\
0 & 0 & 0 & -\zeta_{1} \\
0 & 0 & -1 & \left(1-Y_{1}\right) Y_{C H 4} \zeta_{1} \\
0 & \zeta_{4} & 0 & -1
\end{array}\right], \\
& \tilde{B}_{i}=\left[\begin{array}{cc}
-\alpha \zeta_{1} & 0 \\
-\zeta_{2} & \zeta_{3} \\
0 & 0 \\
0 & 0
\end{array}\right], \quad \tilde{C}=\left[\begin{array}{cccc}
1 & 0 & 0 & 0 \\
0 & 1 & 0 & 0 \\
0 & 0 & 1 & 0
\end{array}\right] \text {. }
\end{aligned}
$$

Note that the matrices of (41) are not unique and different representations can be obtained by considering different arrangements of the scheduling variables.

The eight modes of (41) are given by evaluating $\tilde{A}_{i}$ and $\tilde{B}_{i}$ over the operation ranges of $\zeta_{i}$. State matrices are not displayed here due to space limitations.

To validate the D-LPV model, the following conditions are considered:

$$
\tilde{x}(0)=\left[\begin{array}{llll}
0.523 & 0.345 & 0.00001 & 0.001
\end{array}\right]^{T} .
$$

The simulated inputs are shown in Fig. 1.
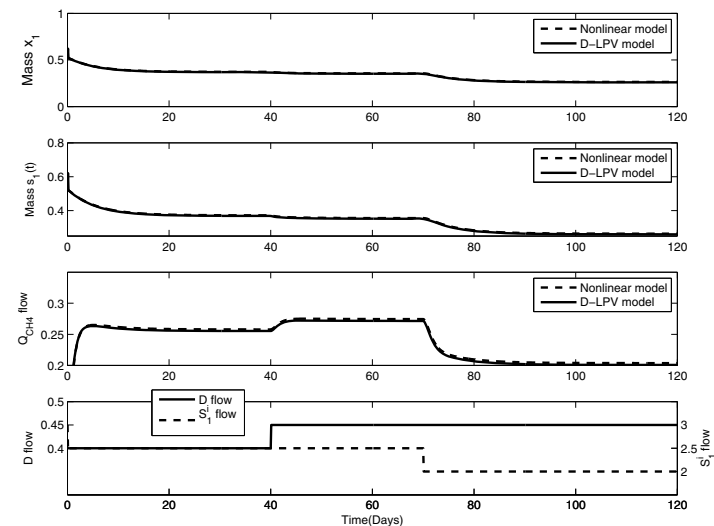

Fig. 1. Nonlinear system outputs and inputs.

Figure 1 displays the comparison between the output signals generated by the D-LPV model and the nonlinear one. The mean-square error for $y_{1}(t), y_{2}$ and $y_{3}(t)$, between the nonlinear and the D-LPV model, is $1.08 \times$ $10^{-5}, 3.45 \times 10^{-4}$ and, $6.67 \times 10^{-6}$, respectively. It is clear, considering Fig. 11 and the small errors, that the D-LPV model matches the nonlinear model.

4.1. State and sensor fault estimation. In order to perform fault estimation, the LPV observer as described in (6) becomes

$$
\begin{aligned}
\dot{z}(t) & =\sum_{j=1}^{8} \rho_{i}(\hat{\zeta}(t))\left[N_{j} z(t)+G_{j} u(t)+L_{j} y(t)\right], \\
\hat{x}(t) & =z(t)+T_{2} y(t) .
\end{aligned}
$$


In addition, to evaluate its performance and robustness, two simulations scenarios, named Case 1 and Case 2, are considered as follows.

Case 1. The method proposed in our previous work (López-Estrada et al., 2013) addressed the observer design without consider disturbances as represented in the system A1. In such a case, robustness is not guaranteed in the presence of noise and disturbance, which can affect also the observer performance as will be detailed below. Note that, despite the same observer structure, the sufficient conditions to compute the observer gains, expressed with the LMIs from Theorem A1 and Corollary 1, are different due to the exclusion of matrices $B_{d}$ and $D_{d}$. Appendix summarizes and describes the sufficient conditions considered in this case.

Case 2. For the system 411, the observer gains for the fault estimation observer (42), which is robust to disturbances and the error provided by the unmeasurable scheduling functions, are computed as proposed in this paper by solving Corollary 1

For both the cases, the following disturbance and noise matrices are considered:

$$
B_{d}=\left[\begin{array}{l}
0 \\
0 \\
1 \\
1
\end{array}\right], \quad D_{d}=\left[\begin{array}{l}
0.7 \\
0.2 \\
0.5
\end{array}\right]
$$

Gain matrices and attenuation levels are computed using Theorem A1 and Corollary 11 with the YALMIP toolbox (Lofberg, 2004). Due to space limitations, gain matrices are not displayed here. Attenuation levels are

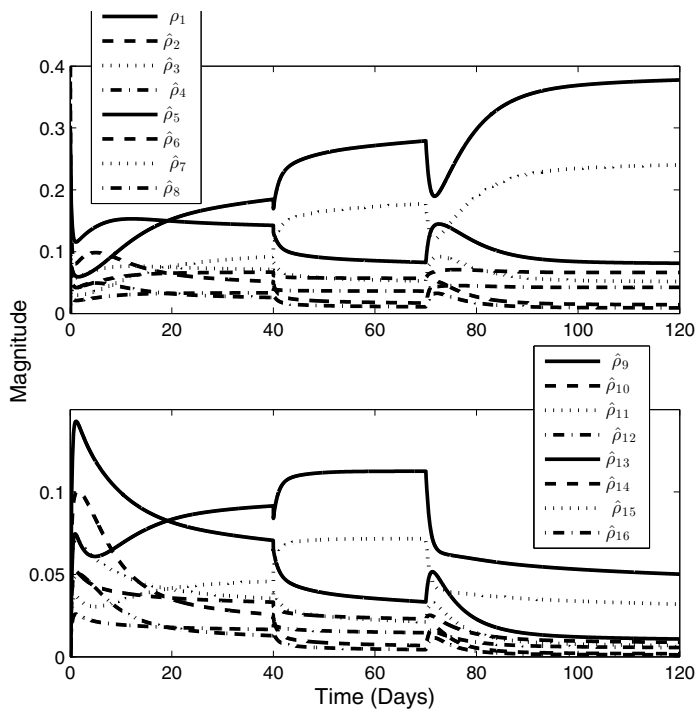

Fig. 2. Estimated gain scheduling functions. $\gamma=0.0024$ and $\gamma=0.000196$, for Case 1 and Case 2, respectively. The small value of $\gamma$ guarantees good attenuation of uncertainties and disturbances.

For both cases the fault estimation observer considers initial conditions $\hat{x}(0)=\left[\begin{array}{lllllll}0 & 0.5 & 1 & 0.4 & -1 & -.2 & 0.3\end{array}\right]$. The disturbance signal included in the system is random signal with a mean of 0.2 and bounded by 0.05 .

Simulation results are displayed as follows. The interactions between the eight models defined by the estimated scheduling functions (40) are displayed in Fig. 2. The quadratic estimation error for Case 1 is displayed in Fig. 3 a) and for Case 2 in Fig. 3 (b). For both the cases, the observer converges fast and with small errors. However, it is clear that, by comparing the error magnitudes, the state observer with observer gains computed by Corollary 1 , which considers additional disturbances attenuation, is better than those obtained in our previous work, where the disturbance vector was not considered. Furthermore, the displayed results also show that in both the cases the observer is still robust to the

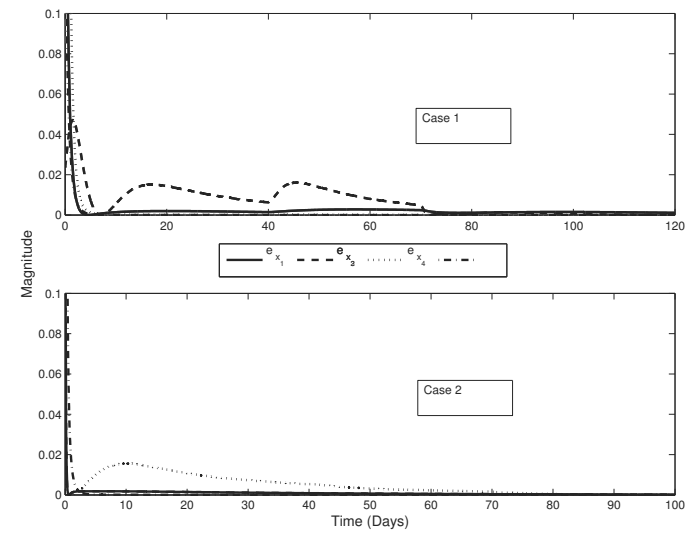

Fig. 3. Quadratic estimation errors between the nonlinear and the D-LPV model: Case 1 (top), Case 2 (bottom).
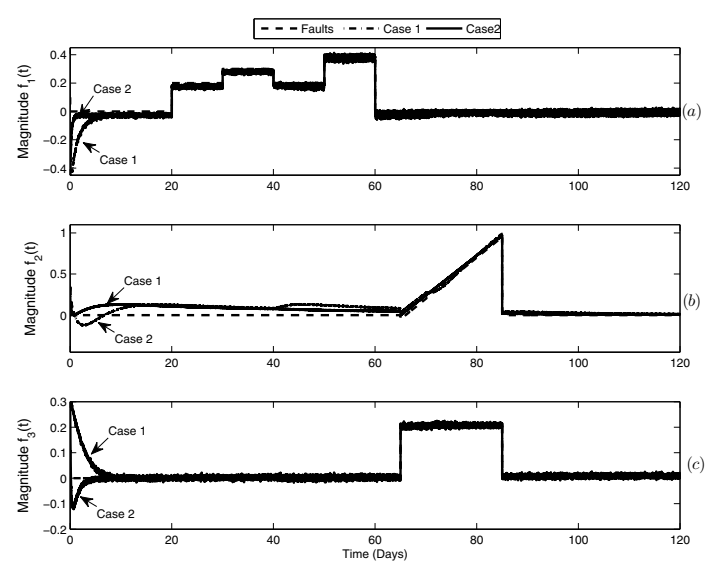

Fig. 4. Fault detection and estimation. 
unmeasurable gain scheduling functions. The real and estimated faults are displayed in Fig. 4 .

The fault on the first sensor is a step-up step-down fault, the one on the second sensor is an incipient fault and that on the third sensor is a one-step fault. The observer estimates faults with good performance, even when they appear simultaneously. The quadratic errors between $f_{1}$ and $\hat{f}_{1}, f_{2}$ as well as $\hat{f}_{2}$ and, $f_{3}$ and $\hat{f}_{3}$ are $0.0064,0.0099$, and 0.00051 for Case 1 and $0.0024,0.0015$, and 0.00025 for Case 2, respectively. The discrepancies between the two cases are mainly caused by the effect of disturbances. It is also clear that, by considering $H_{\infty}$ performance criteria, the error injected by the unmeasurable scheduling functions is well attenuated. Also, as displayed in Fig. 4, by considering disturbance attenuation it is possible to increase the convergence time. For example, the fault 3 converges in three days with Case 2 regardless the eight days of Case 1. Moreover, by considering disturbance attenuation, it is possible to increase reliability and avoid false alarms. For example, for Case 1, the fault estimation from $t=40$ days to $t=50$ days on Sensor 2, which is after the threshold limit, can be interpreted as a false alarm. Of course, this false alarm can be eliminated by predefining different threshold levels or considering a more elaborated adaptive threshold (Montes de Oca et al., 2011). Nevertheless, this problem is beyond the scope of this paper.

\section{Conclusions}

In this paper, a state estimation as well as fault detection, isolation, and sensor fault estimation observer for descriptor-LPV system with unmeasurable gain scheduling functions was proposed. In order to estimate the faults, the states of the D-LPV system were augmented by considering the fault vector as auxiliary state variables such that the augmented vector contains information about the original states and faults. Because the gain scheduling functions of the D-LPV system and the observer are dependent on different time varying scheduling functions, the D-LPV system was transformed into an uncertain one in order to obtain a descriptor error system depending on both the estimated and unmeasurable scheduling functions. Sufficient conditions for the existence of the robust observer were given by a set of linear matrix inequalities.

The applicability and performance of the proposed method was illustrated through an application example of an anaerobic bioreactor. Simulations results also show that the influence of the errors due to the unmeasurable scheduling functions and disturbance were well attenuated due to the $H_{\infty}$ criterion considered. Based on fault estimation, a fault tolerant control scheme could be implement. Additionally, a further extension of these results can be obtained by considering the $H_{-} / H_{\infty}$ approach in order to synthesize an observer sensitive to faults and insensitive to disturbances.

\section{Acknowledgment}

This work was supported by CONACyT (Consejo Nacional de Ciencia y Tecnología), Mexico, and Ministère des Affaires Étrangères, France. The support is gratefully acknowledged.

\section{References}

Aguilera-González, A., Astorga-Zaragoza, C.M. Adam-Medina, M., Theilliol, D., Reyes-Reyes, J. and Garcia-Beltrán, C.-D. (2013). Singular linear parameter-varying observer for composition estimation in a binary distillation column, IET Control Theory \& Applications 7(3): 411-422.

Alcorta-García, E., Saucedo-Flores, S. and Díaz-Romero, D.A (2014). Intelligent fault diagnosis in nonlinear systems, Intelligent Automation and Soft Computing 20(2): 201-212.

Astorga-Zaragoza, C.M., Theilliol, D., Ponsart, J.C. and Rodrigues, M. (2011). Observer synthesis for a class of descriptor LPV systems, American Control Conference, ACC 2011, San Francisco, CA, USA, pp. 722-726.

Blesa, J., Rotondo, D., Puig, V. and Nejjari, F. (2014). FDI and FTC of wind turbines using the interval observer approach and virtual actuators/sensors, Control Engineering Practice 24: 138-155.

Chadli, M., Abdo, A. and Ding, S.X. (2013a). $H_{-} / H_{\infty}$ fault detection filter design for discrete-time Takagi-Sugeno fuzzy system, Automatica 49(7): 1996-2005.

Chadli, M., Aouaouda, S., Karimi, H. and Shi, P. (2013b). Robust fault tolerant tracking controller design for a VTOL aircraft, Journal of the Franklin Institute 350(350): 2627-2645.

Chadli, M. and Darouach, M. (2011). Robust admissibility of uncertain switched singular systems, International Journal of Control 84(10): 1587-1600.

Chen, J. and Patton, R.J. (1999). Robust Model-based Fault Diagnosis for Dynamic Systems, Kluwer Academic Publishers, Norwell, MA.

Ding, S.X. (2008). Model-Based Fault Diagnosis Techniques: Design Schemes, Algorithms and Tools, Springer, Berlin/Heidelberg.

Duan, G.R. (2010). Analysis and Design of Descriptor Linear Systems, Springer, New York, NY.

Frank, P. (1996). Analytical and qualitative model-based fault diagnosis - a survey and some new results, European Journal of Control 2(1): 6-28.

Gertler, J. (1997). Fault detection and isolation using parity relations, Control Engineering Practice 5(5): 653-661.

Hamdi, H., Rodrigues, M., Mechmeche, C. and Braiek, N.B (2012a). Robust fault detection and estimation for descriptor systems based on multi-models concept, International Journal of Control, Automation and Systems 10(6): 1260-1266. 
Hamdi, H., Rodrigues, M., Mechmeche, C. and Theilliol, D. (2012b). Fault detection and isolation for linear parameter varying descriptor systems via proportional integral observer, International Journal of Adaptive Control and Signal Processing 26(3): 224-240.

Hwang, I., Sungwan, K., Youdan, K. and Seah, C. (2010). A survey of fault detection, isolation, and reconfiguration methods, IEEE Transactions on Control Systems Technology 18(3): 636-653.

Ichalal, D., Marx, B., Ragot, J. and Maquin, D. (2010). State estimation of Takagi-Sugeno systems with unmeasurable premise variables, IET Control Theory \& Applications 4(5): 897

Isermann, R. (1984). Process fault detection based on modeling and estimation methods-a survey, Automatica 20(4): 387-404.

Kamidi, R. (2000). Controllability and observability of polytopic linear models, Technical report, Eindhoven University of Technology, Eindhoven.

Lendek, Z., Guerra, T.M., Babuŝka, R. and De Schutter, B. (2011). Stability Analysis and Nonlinear Observer Design Using Takagi-Sugeno Fuzzy Models, Studies in Fuzziness and Soft Computing, Vol. 262, Springer, Berlin/Heidelberg.

Lofberg, J. (2004). A toolbox for modeling and optimization in MATLAB, Proceedings of the Computer Aided Control System Design Conference, Taipei, Taiwan, pp. 284-289.

López-Estrada, F.-R., Ponsart, J., Theilliol, D. and Astorga-Zaragoza, C.-M. (2013). Fault estimation observer design for descriptor-LPV systems with unmeasurable gain scheduling functions, 2nd International Conference on Control and Fault-Tolerant Systems (SYSTOL), Nice, France, pp. 269-274.

López-Estrada, F.R., Ponsart, J.C., Theilliol, D., Astorga-Zaragoza, C.-M. and Aberkane, S. (2014a). Fault diagnosis based on robust observer for descriptor-LPV systems with unmeasurable scheduling parameters, 19th World Congress of the International Federation of Automatic Control, Cape Town, South Africa, pp. 1079-1084.

López-Estrada, F.-R., Ponsart, J., Theilliol, D. and Astorga-Zaragoza, C.-M. (2014b). Robust sensor FDI observer for polytopic descriptor-LPV systems with unmeasurable scheduling varying parameter, American Control Conference, Portland, OR, USA.

Lunze, J., Blanke, M., Staroswiecki, M. and Kinnaert, M. (2006). Diagnosis and Fault-Tolerant Tolerant Control, Springer, Berlin/Heidelberg.

Martínez-Sibaja, A., Astorga-Zaragoza, C.M., Alvarado-Lassman, A., Posada-Gómez, R., Aguila-Rodríguez, G., Rodríguez-Jarquin, J. and Adam-Medina, M. (2011). Simplified interval observer scheme: A new approach for fault diagnosis in instruments, Sensors 11(1): 612-622.

Masubuchi, I., Kato, J., Saeki, M. and Ohara, A. (2004). Gain-scheduled controller design based on descriptor representation of LPV systems: Application to flight vehicle control, 43rd IEEE Conference on Decision and Control, Atlantis, Paradise Island, The Bahamas, Vol. 1, pp. 815-820.

Montes de Oca, S., Puig, V. and Blesa, J. (2011). Robust fault detection based on adaptive threshold generation using interval LPV observers, International Journal of Adaptive Control and Signal Processing 26(3): 258-283.

Nagy-Kiss, A.M., Marx, B., Mourot, G., Schutz, G. and Ragot, J. (2011a). Observers design for uncertain Takagi-Sugeno systems with unmeasurable premise variables and unknown inputs. application to a wastewater treatment plant, Journal of Process Control 21(7): 1105-1114.

Nagy Kiss, A.M., Marx, B., Mourot, G., Schutz, G. and Ragot, J. (2011b). State estimation of two-time scale multiple models. Application to wastewater treatment plant, Control Engineering Practice 19(11): 1354-1362.

Samy, I., Postlethwaite, I. and Gu, D.-W. (2011). Survey and application of sensor fault detection and isolation schemes, Control Engineering Practice 19(7): 658-674.

Theilliol, D. and Aberkane, S. (2011). Design of LPV observers with unmeasurable gain scheduling variable under sensors faults, IFAC World Congress, Milan, Italy, pp. 7613-7618.

Wang, J.L., Yang, G.-H. and Liu, J. (2007). An LMI approach to index and mixed fault detection observer design, Automatica 43(9): 1656-1665.

Xu, S. and Lam, J. (2006). Robust Control and Filtering of Singular Systems, Springer, Berlin/Heidelberg.

Yoneyama, J. (2009). $H_{\infty}$ filtering for fuzzy systems with immeasurable premise variables: An uncertain system approach, Fuzzy Sets and Systems 160(12): 1738-1748.

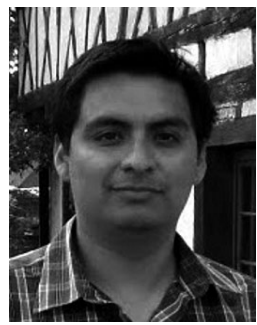

Francisco Ronay López-Estrada received his Ph.D. in automatic control from the University of Lorraine, France, in 2014. He has been with Instituto Tecnológico de Tuxtla Gutiérrez, Mexico, as an assistant professor since 2008. He received his M.Sc. degree in electronic engineering in 2008 from Centro Nacional de Investigación y Desarrollo Tecnológico (CENIDET), Mexico. His research interests are in descriptor systems, linear parameter varying systems, fault detection, fault tolerant control systems, and process control.

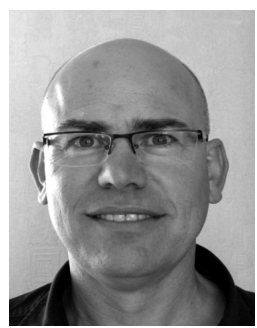

Jean-Christhope Ponsart received his Ph.D. in 1996 from the University of Savoie in Annecy, France, in nonlinear control of magnetic suspensions and its digital implementation aspects. In 1997, he participated in the design and implementation of real-time controllers with a digital signal processor architecture for an industrial company. He has been with the Research Center for Automatic Control of Nancy (CRAN), France, as an assistant professor since 1998. His current interests include fault diagnosis and accommodation, and fault tolerant control. 


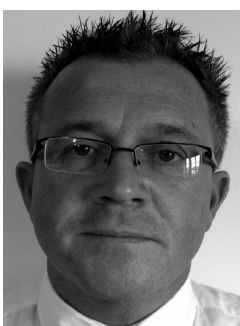

Didier Theilliol received the $\mathrm{Ph} . \mathrm{D}$. degree in control engineering from Nancy University, France, in 1993. Since 2004, he has been a full professor in the Research Centre for $\mathrm{Au}$ tomatic Control of Nancy (CRAN), University of Lorraine, where he coordinates and leads national, European, and international R\&D projects in steel industries, waste-water treatment plans or the aerospace domain. His current research interests include model-based fault diagnosis (FDI) method synthesis and active fault-tolerant control (FTC) system design for LTI, LPV, multi-linear systems, and also reliability analysis. Professor Theilliol has published over 100 journal/conference papers and is a coauthor of the book Fault-tolerant Control Systems: Design and Practical Applications.

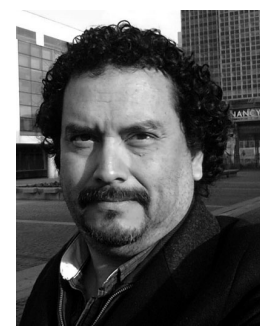

Carlos Manuel Astorga-Zaragoza was born in Minatitlán, Veracruz, Mexico, in 1967. He received the M.Sc. degree in electronic engineering from Centro Nacional de Investigación y Desarrollo Tecnológico (CENIDET), Cuernavaca, Morelos, Mexico, in 1993, and the Ph.D. in process engineering from Université Claude Bernard Lyon 1, France, in 2001. Since 1993, he has held teaching and research positions at CENIDET, where he mainly works on automatic control of energy conversion processes. His research interests include the nonlinear observers, fault diagnosis, and control systems with applications, in particular to process engineering.

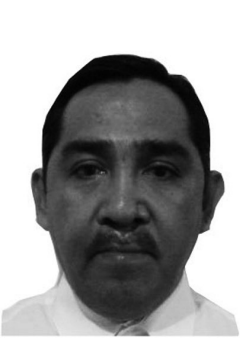

Jorge-Luis Camas-Anzueto received the Ph.D. degree from Instituto Nacional de Astrofísica Óptica y Elctrónica (INAOE), Puebla, Mexico, in 2004. He is currently a researcher at Instituto Tecnológico de Tuxtla Gutiérrez, Chiapas, Mexico. His research interests include optical sensors, fiber sensors, and optoelectronics control.

\section{Appendix}

\section{Synthesis of the fault estimation observer}

Consider a descriptor LPV system under sensor faults given by

$$
\begin{aligned}
\tilde{E} \dot{\tilde{x}}(t) & =\sum_{i=1}^{h} \rho_{i}(\tilde{x}(t))\left[\tilde{A}_{i} \tilde{x}(t)+\tilde{B}_{i} u(t)\right], \\
y(t) & =\tilde{C} \tilde{x}(t)+f(t) .
\end{aligned}
$$

Under the assumptions of admissibility and $\mathrm{R} / \mathrm{I}$-observability, the following LPV observer is proposed to estimate states and faults:

$$
\begin{aligned}
\dot{z}(t) & =\sum_{j=1}^{h} \rho_{j}(\hat{x}(t))\left[N_{j} z(t)+G_{j} u(t)+L_{j} y(t)\right], \\
\hat{x}(t) & =z(t)+T_{2} y(t) .
\end{aligned}
$$

The following result guarantees state and fault estimation.

Theorem A1. (López-Estrada et al., 2013) Given the system (Al), let the attenuation level satisfy $\gamma>0$. Then the observer (A2) exists if (AT) is stable and $\left\|G_{r \bar{f}}(s)\right\|_{\infty}<\gamma$ and if there are matrices

$$
P=\left[\begin{array}{cc}
P_{1} & 0 \\
0 & P_{2}
\end{array}\right],
$$

$P_{1}=P_{1}^{T}>0, P_{2} \geq 0$ and gain matrices $Q_{j}=P_{1} K_{j}$ such that $\forall i, j \in\{1,2, \ldots, h\}$ :

$$
E^{T} P_{2}=P_{2}^{T} E \geq 0
$$

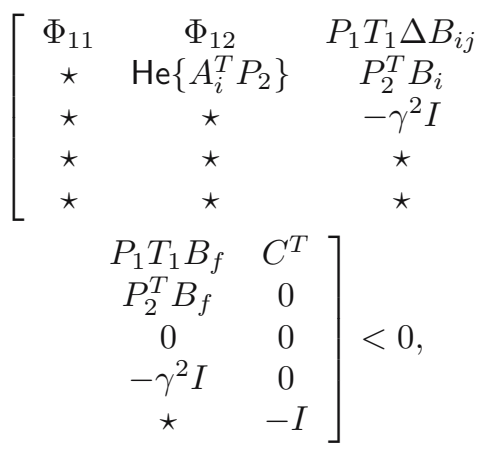

with

$$
\begin{aligned}
& \Phi_{11}=\operatorname{He}\left\{\left(T_{1} A_{j}\right)^{T} P_{1}+Q_{j} C\right\}, \\
& \Phi_{12}=\left(T_{1} \Delta A_{i j}\right)^{T} P_{1} .
\end{aligned}
$$

The proof is omitted and can be consulted in the cited paper. It is clear that Theorem A1 guaranteed asymptotic stability on the estimation error but not robustness to disturbances.

Received: 14 March 2014

Revised: 2 August 2014 